\title{
Performance Evaluation of THT Deployment with Different Scheduling Methods in WSN
}

\author{
Anu Rathee \\ Department of Computer Science Technology \\ Manav Rachna International University, \\ Faridabad, India
}

\begin{abstract}
This paper investigates the performance of a new node deployment model named Tri -Hexagon-Tiling for different types of scheduling in terms of energy consumption. This paper includes three types of scheduling methods by which we can allocate the time slots to the network nodes in order to assign them the periods of activity and inactivity. All the simulations have been conducted in OMNeT++ [1] discrete event simulator. This paper investigates the energy composition related to different scheduling methods to provide a guideline for the best choice of certain method according to the application scenario.
\end{abstract}

Keywords: Wireless sensor networks, THT deployment, $X$ scheduling, V scheduling, Leon Cross scheduling, Energy consumption

\section{INTRODUCTION}

The protocol divides the sensor networks into time zones.During the initialization phase, the gateway broadcasts an initial SYNC packet to the neighboring nodes. The nodes receiving the initialization message are categorized as being in first time zone. The nodes then synchronize their internal clock, set their time zone, and MERLIN adopts multicast upstream and multicast downstream transmission to relay information both to the gateway and away from it. Time zone updates and exchange of synchronization packets happens through periodic local broadcast. All nodes share a common sensing task; hence there is a sensing redundancy. This employs that not all the sensors are required to perform the sensing task during the whole system lifetime. So it is important to schedule the nodes. The concept of scheduling comes from the operating systems. Round Robin is one of the simplest scheduling algorithms for processes in the operating systems, which assigns time slices to each process in equal portions and in order, handling all processes without priority. In networks processes may be replaced by nodes [4].

A proper schedule not only avoids collisions by silencing the interferes of every receiver node in each time slot but also minimizes the number of time slots hence the latency: The larger latency may require a higher data rate and hence higher energy consumption [5].

The aim of the scheduling is to allocate the timeslots to the network nodes in order to assign them the periods of activity and inactivity. Scheduling helps in synchronization of the neighboring nodes for the transmission and reception of packets. Typically, Data patterns can be classified in continuous and discontinuous. Continuous data patterns can forward a packet without interruption from source to destination, while discontinuous data patterns introduce some delay in forwarding process. This delay can be used to avoid collisions or save energy. A packet traveling on a continuous data pattern can reach the destination without interruption. Alternatively, in discontinuous data pattern, a packet is forwarded after a certain delay towards the destination or away from it. An appropriate scheduling policy can be developed according to such expected data patterns [6].

\section{LITERATURE REVIEW}

In 2004, Barbara Hohlt et al., proposed a distributed on-demand power-management protocol for collecting data in sensor networks. Flexible Power Scheduling allowed exploitation of the natural energy conservation inherent in slotted time division schemes. In the paper, they presented a dynamic distributed time division scheduling protocol that facilitated power management by enabling nodes to turn off their radios during idle slots.

A.G. Ruzzelli et al., in 2005, has investigated the use of intelligent agents in the delivery of adaptively at the networking layers. This is achieved by using two preexisting technology sets developed in part by the authors; these are: the energy-efficient integrated MERLIN protocol and Agent Factory a rapid prototyping environment for agent deployment. Three instruments facilitate this: the provision of two efficient and interchangeable scheduling tables; the ability to generate virtual network sectors; the adoption of autonomous mobile agents.

Antonio G. Ruzzelli et al., in 2005 , addressed the tradeoff between energy conservation and latency. In particular they contrasted the $\mathrm{X}$ and $\mathrm{V}$ scheduling family schemes with respect to the following properties: network setup time, network lifetime and message latency. The work showed a better performance of the $\mathrm{X}$ scheduling than the $\mathrm{V}$-scheduling in terms of latency of messages and network setup time. It was concluded that the $\mathrm{X}$ scheduling should be used for applications in which some energy can be traded off for a decrease of latency of messages and for applications in which latency is a tighter constraint. In contrast, the $\mathrm{V}$-scheduling performs better than the X-scheduling in terms of percentage of collisions and network lifetime. As a result the V-scheduling was found to be more suitable for low data traffic applications where the need of saving energy was of paramount importance.

\section{THT DEPLOYMENT MODEL}

A tiling is the covering of the entire plane with figures which do not overlap nor leave any gaps. Tilings are also sometimes called tessellations. Among different tilings we use a semiregular tiling (which has exactly eight different tilings) where every vertex uses the same set of regular polygons. A regular polygon has the same side lengths and interior angles. We consider a semi-regular tiling that uses triangle and hexagon in the two dimensional plane, the so-called 3-6-3-6 Tri-Hexagon Tiling. Here we combine the advantages of a triangle grid and a hexagon grid. 


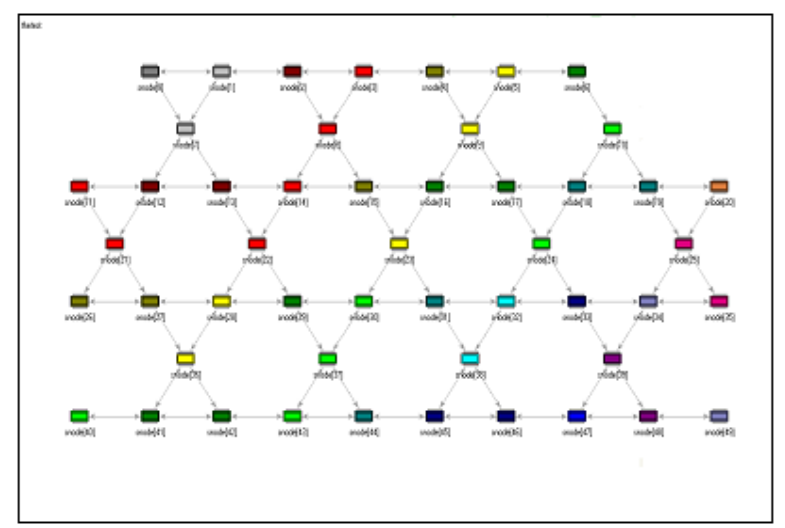

Fig.1The network diagram of THT deployment

\section{SCHEDULING METHODS}

The deployment model adopts a time zone based transmission scheduling which allows the nodes in the same time zone to use the same slot to transmit. The scheduling table is usually transmitted by the gateway during the initialization phase, and schedules the periods of nodes activity and inactivity. Variation of either slot length or frame time causes different delay in packet scheduling. The total length of the table is equal to the length of a single frame while each small rectangle represents a time slot. In the tables, the nodes within the same time zone contend the channel for transmission, the adjacent zone owns the slot for reception and the nodes in the further time zones are in sleep mode. The tables represent the models of inter-zone scheduling that provide different priorities to energy consumption

\subsection{Scheduling}

In $\mathrm{X}$ scheduling, nodes remain active most of the time and hence higher channel utilization is achieved here. They can hold concurrent transmission slots between nearby time zone. Scheduling of further zones can achieved by appending the same table.

$\mathrm{X}$ table performs the upstream and downstream concurrent transmission by forwarding a packet to 8 time zones towards the gateway or in opposite direction within the same frame time. $\mathrm{X}$ table allocates 8 timeslots for upstream, 8 timeslots for downstream and 1 timeslot for local broadcast. The color of the node depicts the zone of the node.

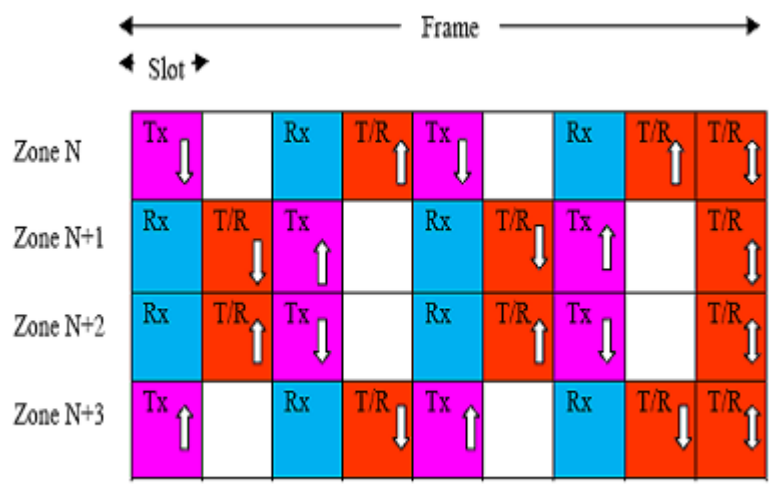

Fig.2: The table of $\mathrm{X}$ scheduling

\subsection{Scheduling}

In this scheduling we have not considered a dedicated slot for local broadcast. The frame is divided into 8 slots. Half of the slots are allocated to downstream data traffic and remaining half slots are allocated to upstream data traffic.

This division of traffic allocation helps avoiding interference between the upstream and downstream data traffic. Figure 1 shows the snapshot of simulated sensor network for Tri Hexagon Tiling deployment for V scheduling.

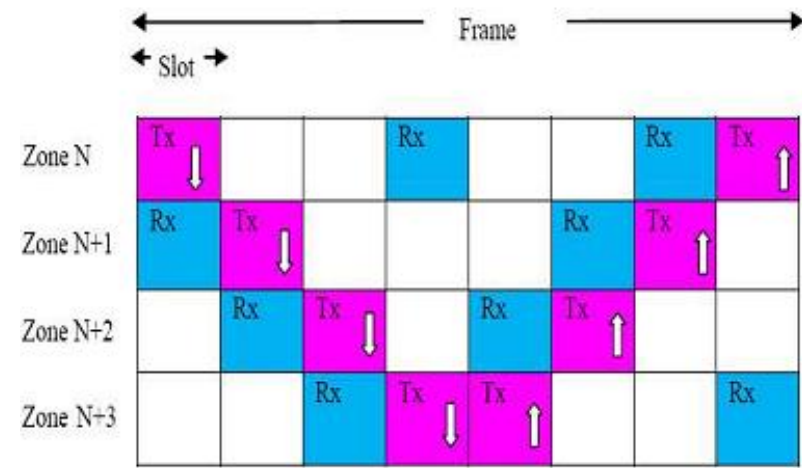

Fig.3: The table of $\mathrm{V}$ scheduling

Figure 3 shows the $\mathrm{V}$ scheduling. In this scheduling we have not considered a dedicated slot for local broadcast. The frame is divided into 8 slots. Half of the slots are allocated to downstream data traffic and remaining half slots are allocated to upstream data traffic.

\subsection{Leon (4x4) Cross Shifted Scheduling}

The Figure 4 shows the Leon $(4 \times 4)$ Crossed Shifted scheduling. In this scheduling, a frame has been divided into 4 slots. Transmission, reception and the local broadcast are done simultaneously. In this scheduling, as soon as the node finishes transmission or reception of the packet it goes into sleep mode. It performs the concurrent upstream and downstream data transmission.

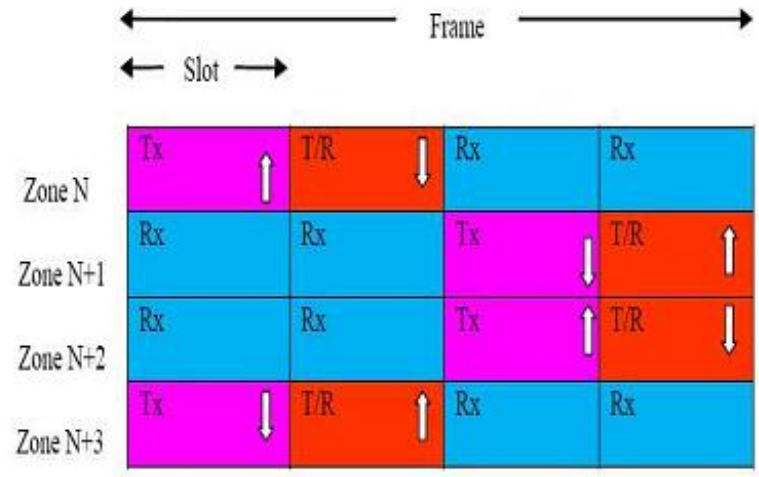

Fig 4 The table of Leon( $(4 \times 4)$ Crossed Shifted Scheduling 


\section{RESULTS AND DISCUSSIONS}

The results are collected by applying all the types of scheduling algorithms to a particular chipset over the increasing extra payload. We collected the results for the chipset TR1001. Figure 5, shows the Energy Tx for the chipset TR1001 for the all scheduling tables. For THT deployment all scheduling methods consumes same amount of energy during transmission.

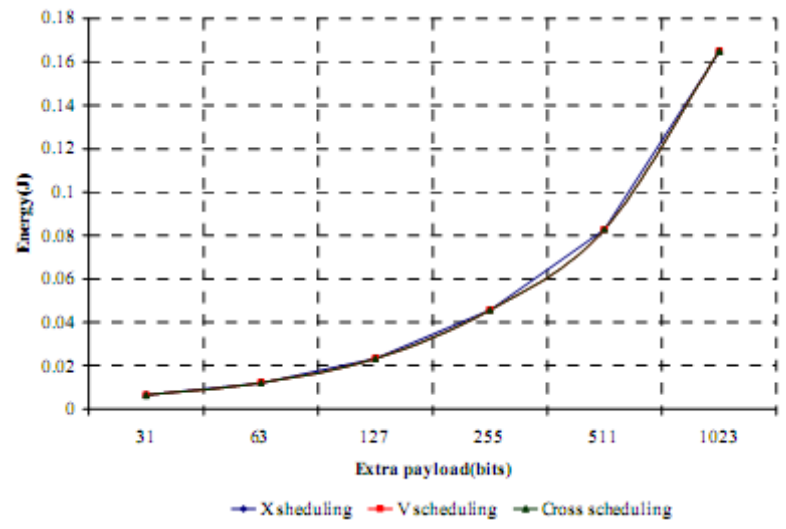

Fig 5: The Energy Tx for Chip TR1001 for various types of scheduling

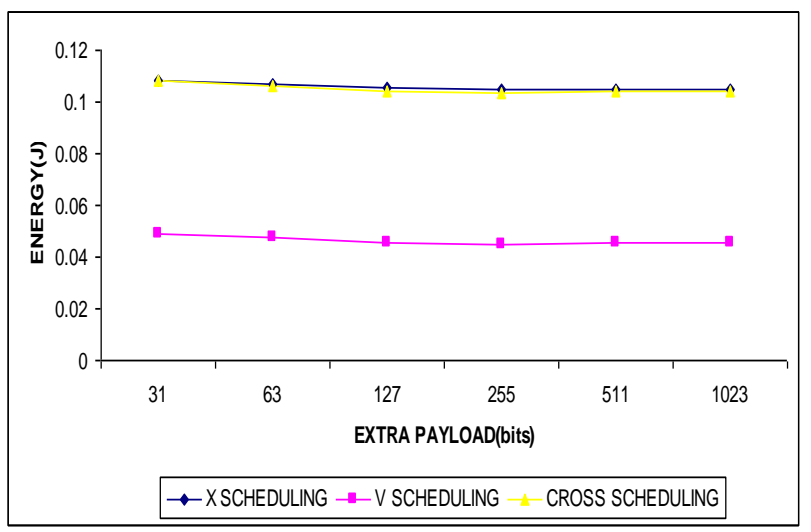

Fig . 6: The Energy Rx for Chip TR1001 for various types of scheduling

Fig.6 shows energy reception for the chipset TR1001 for all scheduling for THT deployment .It depicts that V scheduling consumes minimum energy while $\mathrm{X}$ scheduling consumes maximum energy up to an extra payload of 511 bits after which cross shifting scheduling overtakes it.

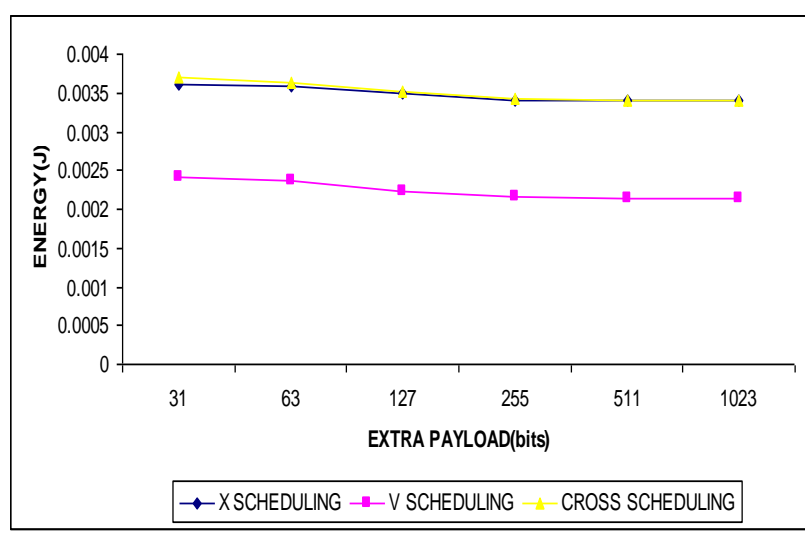

Fig. 7: The Energy switching for Chip TR1001 for various types of scheduling

Figure 7 shows the energy consumption by the chipset TR1001 during switching from one state to another, for various types of scheduling for THT deployment. It is observed that V scheduling consumes minimum energy gradually decreases up to the extra payload of 255 bits after that it becomes stable and constant. X scheduling varies from $0.003619 \mathrm{~J}$ to $0.0034147 \mathrm{~J}$ of energy which is maximum among all the scheduling consumption and remains to $0.0034 \mathrm{~J}$.

Figure 8 shows the energy consumption during sleep by the transceiver TR1001 for all type of scheduling method .It is observed that $\mathrm{V}$ scheduling consumed maximum energy than $\mathrm{X}$ $\&$ Cross Scheduling.

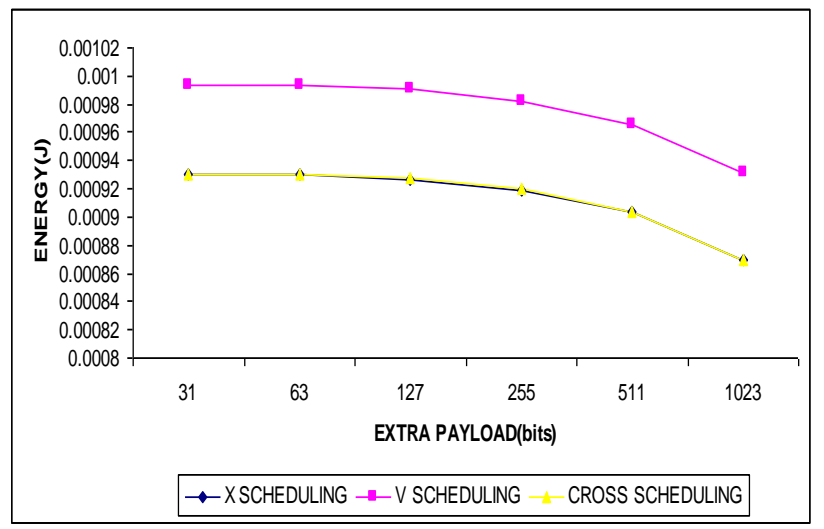

Fig. 8: The Energy Sleep for Chip TR1001 for various types of scheduling

Figure 9 shows the total energy consumption by the chipset TR1001 for different scheduling for tri-hexagon tiling deployment. It is observed that $\mathrm{V}$ scheduling consumes minimum energy and $\mathrm{X}$ and crossed shifted scheduling consumes same amount of maximum energy.

Table 1 shows the Energy Tx, Energy Rx, Energy Switching, Energy Sleep and Total Energy for the THT distribution for chipset TR1001 at 511 bits of extra payload. We can see that at 


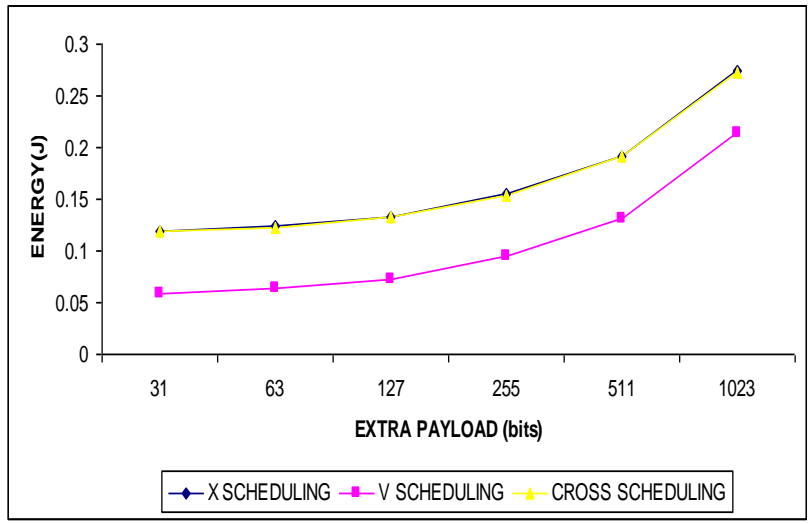

Figure 9: The Total Energy for Chip TR1001 for various types of scheduling

the 511bits of extra payload energy required for the reception of data and energy consumed during transition from one state to another is lower for THT deployment in V scheduling. Overall in the chipset total energy consumed in case of $\mathrm{V}$ scheduling is less as compared to other scheduling methods due to the fact that energy is less consumed in hexagonal arrangement of nodes, through the few nodes that come in the path to reach to the gateway and hence, less energy consumption.

Table .1: Energy consumption of THT deployment for three types of scheduling at extra payload of 511 bits.

(a)

\begin{tabular}{|l|l|l|l|l|l|l|}
\hline \multirow{2}{*}{ Chipset } & \multicolumn{3}{|l|}{ Energy Tx (J) } & \multicolumn{3}{l|}{ Energy Rx (J) } \\
\cline { 2 - 7 } & $\mathrm{X}$ & $\mathrm{V}$ & Cross & $\mathrm{X}$ & $\mathrm{V}$ & Cross \\
\hline TR1001 & 0.082 & 0.082 & 0.0828 & 0.104 & 0.045 & 0.1039 \\
\hline
\end{tabular}

(b)

\begin{tabular}{|l|l|l|l|l|l|l|}
\hline \multirow{2}{*}{ Chipset } & \multicolumn{3}{|l|}{ Energy sleep (J) } & \multicolumn{3}{|c|}{ Energy Switching (J) } \\
\cline { 2 - 7 } & $\mathrm{X}$ & $\mathrm{V}$ & Cross & $\mathrm{X}$ & $\mathrm{V}$ & Cross \\
\hline TR1001 & $\begin{array}{l}0.000 \\
9\end{array}$ & $\begin{array}{l}0.000 \\
97\end{array}$ & 0.0009 & 0.003 & 0.002 & 0.003 \\
\hline
\end{tabular}

(c)

\begin{tabular}{|l|c|c|c|}
\hline \multirow{2}{*}{ Chipset } & \multicolumn{3}{|c|}{ Total Energy (J) } \\
\cline { 2 - 4 } & $\mathrm{X}$ & $\mathrm{V}$ & Cross \\
\hline TR1001 & 0.19182 & 0.13156 & 0.19112 \\
\hline
\end{tabular}

\section{CONCLUSIONS}

Packet flow is achieved through a division of the network in time zones and through the usage of appropriate transmission scheduling. In particular, 3 scheduling methods, namely $\mathrm{X}$ tables, V table, Crossed Shifted have been proposed and have been evaluated for the energy consumption. These scheduling tables can be used in different network scenarios.
It is inferred from the above concluded results that the energy consumption depends on the mode of radio i.e. transmitting, receiving, sleep or switching; type of scheduling i.e. $\mathrm{X}$ scheduling, V scheduling, Crossed Shifted scheduling and the way the sensor nodes are deployed in the wireless sensor networks i.e. Tri-Hexagon- tiling deployment. The above discussed inferences could help in making a proper choice for energy efficient deployment of sensor networks.

In the reception mode, $\mathrm{V}$ scheduling consumes minimum energy for the chipset TR1001. V scheduling consumes minimum energy for state transitions for all chipsets. For the chipset TR1001 Crossed Shifted scheduling and X scheduling consumes minimum energy during sleep mode. Overall, V scheduling consumes minimum total energy for the chipset TR1001in the THT deployment model.

\section{REFERENCES}

[1] Antonio G. Ruzzelli, "Wireless Sensor Networks:Enhancing Performance through Integration of MAC and Routing Protocols",A Thesis submitted to University College Dublin for the degree of $\mathrm{PhD}$ in the College of Engineering, Mathematical and Physical Sciences.

[2] S. Ray et al., Robust location detection in emergency sensor networks, IEEE INFOCOM 2003, 2, 1044-1053, March 2003.

[3] Y. Zou and K. Chakrabarty, Sensor deployment and target localization based on virtual forces, IEEE INFOCOM 2003, 2, 1293-1303, San Francisco, 2003.

[4] Antonio G. Ruzzelli, Richard Tynan, and G.M.P. O" Hare. An Energy-Efficient and Low-Latency Routing Protocol for Wireless Sensor Networks. In Proceedings of the Systems Communications, ICW, pages 449-454, August 2005.

[5] Chih-Yung Chang, Kuei-Ping Shih and Shih-Chieh Lee, “ ZBP: A Zone Based Broadcasting Protocol for Wireless Sensor Networks". Wireless Personal Communication (2005)33:53-68.

[6] A.G. Ruzzelli, M.J. O" Grady, G.M.P. O" Hare and R. Tynan. "Adaptive scheduling in Wireless Sensor Networks", In proceeding of WAC2005, the 2nd IFIP Workshop on Autonomic Communication Athens, Greece, LNCS press, 2005.

[7] Sinem Coleri Ergen and Pravin Varaiya, “ TDMA Scheduling Algorithms for Sensor Networks”, July 2005.

[8] Shubham Jain and Sanjay Srivastava, “ A A Survey and Classification of Distributed Scheduling Algorithms for Sensor Networks", 2007 IEEE.

[9] Yunali Wang, Xianghui Liu, Jianping Yin, Ning Jing, "Efficiently Link Scheduling in Wireless Sensor Network", Submitted in 20061 st International Symposium on Pervasive Computing and Applications. 